\title{
Increasing penetration of renewals in isolated power systems using energy storage systems
}

\author{
L. Rouco ${ }^{1}$, I. Azpiri ${ }^{2}$, I. Gómez de $\mathrm{Olea}^{2}$ and J. Tabernero ${ }^{1}$ \\ ${ }^{1}$ Universidad Pontificia Comillas \\ Calle de Alberto Aguilera, 23, 28015 Madrid, Spain \\ ${ }^{2}$ Iberdrola Renovables \\ Parque Empresarial Cristalia, Edificio-9, Vía de los Poblados, 3, 28033 Madrid, Spain
}

\begin{abstract}
Frequency stability is one of the most relevant issues in operation of isolated power systems. High penetration of renewals may affect significantly frequency stability of isolated power systems since wind and solar photovoltaic generation have neither inertia nor primary frequency regulation. An alternative is the use of storage systems in the most critical operating conditions in such a way that if a generator trips, storage systems are subsequently tripped and the power balance is restored. This paper presents an approach to determine the maximum renewable generation in an isolated power system to prevent non-admissible frequency excursions in case of generator tripping. Moreover, such approach allows sizing an energy storage system to increase the penetration of renewals.
\end{abstract}

Keywords. Isolated power systems, Energy storage systems, Frequency stability

\section{Introduction}

The most relevant issue in operation of isolated power systems is frequency stability [1]. Frequency stability is concerned with the ability of the generators to supply the loads within acceptable frequency ranges in case of generator tripping. Frequency stability is governed by the kinetic energy stored in the generator-prime mover rotating masses and the prime mover frequency primary regulation. If frequency excursions are not within $+/-2.5$ $\mathrm{Hz}$ range (see [2]), cascade tripping of the remaining generators can occur because of generator over/under frequency protections tripping. High penetration of renewals may affect significantly frequency stability of isolated power systems since wind and solar photovoltaic generation have neither inertia nor primary frequency regulation. An alternative is the use of storage systems in the most critical operating conditions in such a way that if a generator trips, storage systems are subsequently tripped and the power balance is restored. The paper determines the critical operating conditions (maximum admissible renewable generation) and the renewable generation to be compensated.

\section{Isolated Power Systems}

Isolated power systems and interconnected power systems exhibit different features. Isolated systems are much smaller than interconnected systems. In addition, they cannot count with the support of the neighbour systems. That is, the size and the lack of external support make isolated systems more vulnerable than interconnected systems. It is especially true in case of disturbances. Hence, the system stability is at risk.

Power system stability is concerned with the ability of the generators to run in synchronism and to supply the loads at acceptable frequency and voltage ranges in case of normal (load variations) and abnormal disturbances (faults, generator tripping) that may occur in power systems. The power system stability problem is a very difficult one. Its study is facilitated by separating it into three subproblems: angle, frequency and voltage stability [3]. We will concentrate on the frequency stability problem since it will be present in all isolated power systems not matter how strong the power network is. Precisely, frequency stability analyzes the capability of generators to supply load at acceptable frequency ranges in case of generator tripping. Frequency results from the generator rotor speeds. Generator rotor speeds result from the equilibrium between the power supplied by their primer movers (either turbines or engines) and the power consumed by the loads. Frequency stability is governed by the inertia of the rotating masses of primer movers and generators and the gain and time constant of the primary frequency regulation of prime movers in such a way that:

- After a generator trips, frequency decays with a rate of change that depends of the inertia of prime movergenerator rotating masses and the magnitude of the generation lost.

- Prime mover primary frequency regulation reacts to the frequency decay increasing the output of the power supplied by the prime movers.

- Frequency stabilizes if two conditions are fulfilled: the remaining on line generators have enough reserve to supply the generation lost and they are also able to increase the power output fast enough to avoid that frequency is below the settings of generator underfrequency protections to avoid generator cascade tripping.

Frequency stability is at risk in isolated power systems because of the fact that the frequency rate of change in case of generator tripping is bigger than in an interconnected power system. The inertia or the kinetic energy of the rotating masses of an interconnected system is much bigger than the inertia of the rotating masses of an isolated system. In addition of, the magnitude of the 
generation that can be tripped compared to the total rotating generation is much bigger in an isolated system than in an interconnected one.

\section{Energy Storage Systems}

Storage of electric energy has been sought since the beginning of the development of electric power systems to overcome the technical problems and over costs that result from the non-storageable nature of electric energy and the time variation of the load. Although many storage systems have been and are been explored, pumped storage power plants have been the only practical solutions for massive storage so far. Instances of storage systems explored are:

- superconducting magnetic storage systems,

- supercapacitors,

- flywheels and

- batteries.

Of course, an alternative approach to storage to overcome the overcosts that result from the time variation of the load is demand side management. The massive development of electric cars will create new opportunities for demand side management through distributed storage systems (the electric cars themselves).

Pumped storage power plants have been incorporated to power systems for many years due to two main reasons:

- To optimize the operation of thermal based generation systems taking into account the constraints of nuclear and coal fired power stations.

- To avoid spillage in hydro systems.

Pumped storage power plants can also contribute to the system ancilliary services:

- Active power-frequency control.

- Reactive power-voltage control.

- Black-start.

Moreover, pumped storage power plants when pumping are within the underfrequency loadshedding schemes. Precisely in the Spanish mainland power system [4]:

- $50 \%$ of the pumped storage power plants are tripped if frequency is below $49,5 \mathrm{~Hz}$.

- The remaining $50 \%$ of the pumped storage power plants are tripped if frequency is below $49,3 \mathrm{~Hz}$.

\section{Fundamentals of Frequency Stability}

The frequency stability problem of a generator feeding a load when a generator is tripped can be understood using the block diagram of Figure 1. It contains representations of the dynamics of the generator-prime mover rotating masses and the prime-mover primary frequency regulation. Model of Figure 1 can be just extended to the analysis of an isolated power system assuming that the frequency is uniform (we assume that all generators exhibit the same speed) and assuming the response of the 2 primary frequency regulation of all generators is identical.

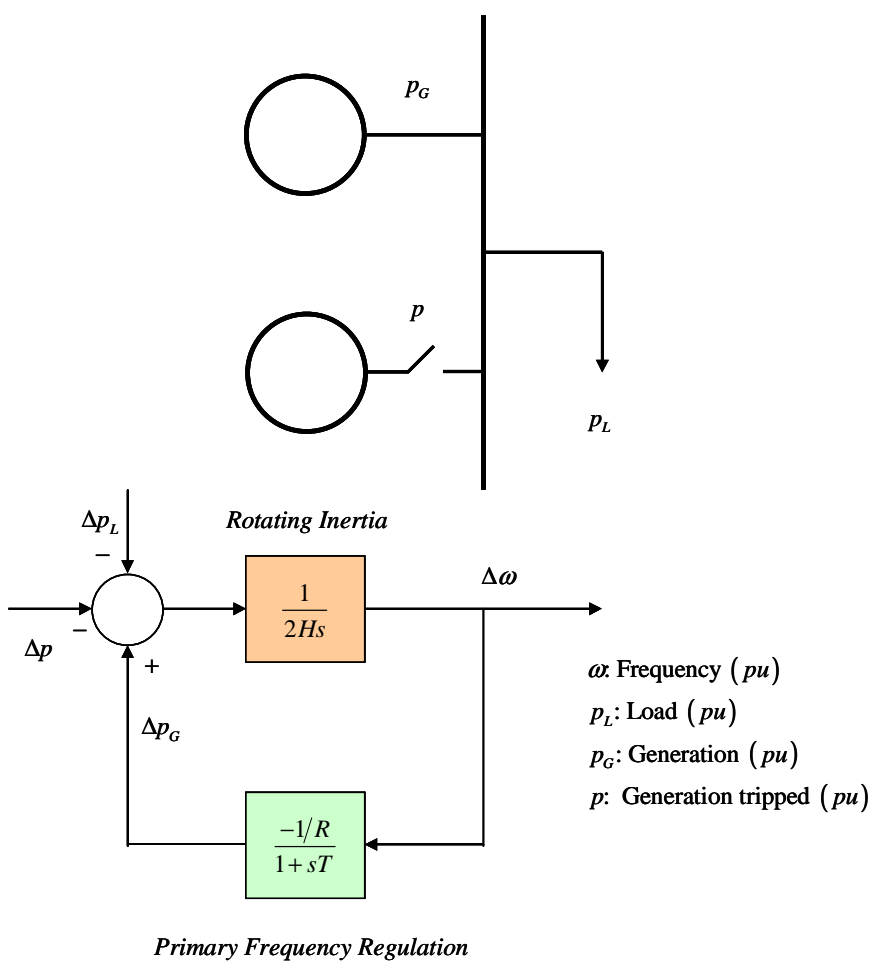

Figure 1: Simplified generator model to study frequency stability.

Equations that describe the block diagram of Figure 1 in the Laplace domain are:

$$
\begin{gathered}
\Delta \omega(s)=\frac{1}{2 H s}\left(\Delta p_{G}(s)-\Delta p_{L}(s)-\Delta p(s)\right) \\
\Delta p_{G}(s)=\frac{-1 / R}{1+s T} \Delta \omega(s) \\
\Delta p(s)=\frac{p}{s}
\end{gathered}
$$

The frequency variation due to generation trip is given by:

$$
\Delta \omega(s)=\frac{-1}{2 H T} \frac{1+s T}{s^{2}+\frac{1}{T} s+\frac{1}{2 H R T}} \frac{\Delta p}{s}
$$

Once the frequency variation has been obtained, the generation variation of on line generation can be determined as:

$$
\begin{aligned}
& \Delta p_{G}(s)=\frac{-1 / R}{1+s T} \Delta \omega(s) \\
& =\frac{1}{2 H R T} \frac{1}{s^{2}+\frac{1}{T} s+\frac{1}{2 H R T}} \frac{\Delta p}{s}
\end{aligned}
$$

The time evolution of the frequency and generation variation in case of a generator tripping is obtained 
computing the Laplace inverse transform of (1) and (2) respectively:

$$
\begin{aligned}
\Delta \omega(t) & =\mathrm{L}^{-1}\{\Delta \omega(s)\} \\
\Delta p_{G}(t) & =\mathrm{L}^{-1}\left\{\Delta p_{G}(s)\right\}
\end{aligned}
$$

Several aspects of the response of the system (the steady state and the transient response) can be discussed using that model. The steady state error of frequency is determined the size of the perturbation and the permanent drop of the primary regulation of the synchronous machine as $\Delta \omega=-R \Delta p$ and the increase of generation is precisely the generation tripped $\Delta p_{G}=\Delta p$. That is:

$$
\begin{aligned}
& \Delta \omega=\lim _{s \rightarrow 0} \Delta \omega(s) s=\lim _{s \rightarrow 0} \frac{-1}{2 H T} \frac{1+s T}{s^{2}+\frac{1}{T} s+\frac{1}{2 H R T}} \frac{\Delta p}{s} s \\
& =\frac{-1}{2 H T} \frac{1}{\frac{1}{2 H R T}} \Delta p=-R \Delta p \\
& \Delta p_{G}=\lim _{s \rightarrow 0} \Delta p_{G}(s) s=\lim _{s \rightarrow 0} \frac{1}{2 H R T} \frac{1}{s^{2}+\frac{1}{T} s+\frac{1}{2 H R T}} \frac{\Delta p}{s} s \\
& =\frac{1}{2 H R T} \frac{1}{\frac{1}{2 H R T}} \Delta p=\Delta p
\end{aligned}
$$

The transient response can be characterized by two variables: the initial rate of change of frequency and the minimum frequency. Both variables are relevant for the design of load shedding schemes. The initial rate of change of frequency is determined by the size of the disturbance and the inertia of the generator-prime mover rotating masses as $d \Delta \omega / d t=-\Delta p / 2 H$.

The maximum frequency deviation can be determined approximately assuming that in case of generator tripping, the frequency decays with constant rate of change and the generator also increased their output with constant rate of change as shown in Figure 2 and Figure 3 [5].
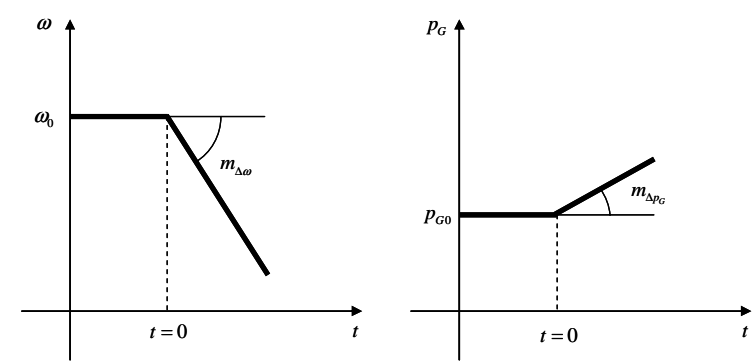

Figure 2: Approximate variation of frequency and generation of a system in case of generation tripping.

In such a case, the differential equation that describes rotor dynamics can be approximated as:

$$
2 H \frac{d \Delta \omega}{d t}=-p+m_{\Delta p_{G}} t
$$

which integration provides an approximation of the frequency variation during the beginning of the transient:

$$
\Delta \omega(t)=\frac{-p}{2 H} t+\frac{1}{4 H} m_{\Delta p_{G}} t^{2}
$$

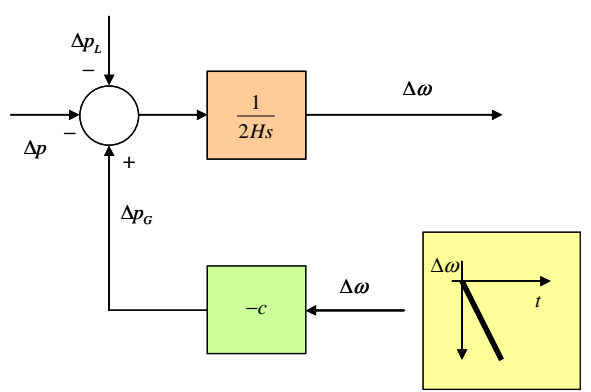

Figure 3: Block diagram representation of the approximate variation of frequency and generation of a system in case of generation tripping.

The maximum frequency deviation is simply obtained making equal to zero the derivative with respect to time of equation (3). The time in which the minimum frequency is reached and the minimum frequency becomes respectively:

$$
\begin{gathered}
t_{f \text { min }}=\frac{p}{m_{\Delta p_{G}}} \\
f_{\text {min }}=f_{0}\left(1-\frac{p}{2 \cdot c}\right)
\end{gathered}
$$

It means that the maximum frequency deviation depends on the size of the disturbance and the speed of the primary frequency regulation of the generators characterized by the parameter $c$ which relates the slope of the generation increase $m_{\Delta p_{G}}$ with the slope of the frequency decay $m_{\Delta \omega}$. Such parameter characterizes the speed of the generator response to a frequency variation. The key parameter of our approximate model to compute the minimum frequency is the factor between the slope of frequency decay and the slope of generation increase. A method to determine such factor for a first order system in proposed.

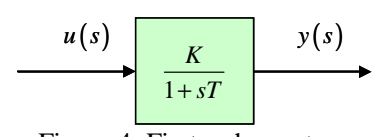

Figure 4: First order system.

If the input $u(t)$ to the first order system of Figure 4 is a ramp of value $r$, the output $y(t)$ will be:

$$
y(t)=r K\left(t-T+T e^{-\frac{t}{T}}\right)
$$


The initial slope is zero whereas the final slope is $K \cdot r$. We are interested in the slope when the time is smaller that the time when the frequency reaches the minimum value. The average value of the slope during that period of time is:

$$
r_{m}=K r \frac{t_{f m i n}-T+T e^{\frac{-t_{\text {fmin }}}{T}}}{t_{f \min }}
$$

where $t_{f m i n}$ is the time when the frequency reaches the minimum value. Therefore, the factor between the generation increase and the frequency decay can be computed as:

$$
c=\frac{r_{m}}{r}=K \frac{t_{\text {fmin }}-T+T e^{-\frac{t_{\text {ffin }}}{T}}}{t_{\text {fmin }}}
$$

Figure 5 displays the frequency variation in an isolated system in case of a generator tripping. Two demand scenarios are considered of a medium size isolated power system (150 and $300 \mathrm{MW})$. A $30 \mathrm{MW}$ generator is tripped. The inertia of the rotating masses is 3 seconds (in system MVA base), the permanent droop of the frequency primary regulation is $0.04 \%$ (in the system MW base) and the time constant of the frequency primary regulation is 1 second. Figure 5 confirms that the initial rate of change, the steady-state error and the minimum frequency depend on the size of the disturbance compared to the total generation. However the time of minimum frequency does not depend on the size of the disturbance. Table I summarizes the relevant magnitudes of the test system in the selected demand scenarios.

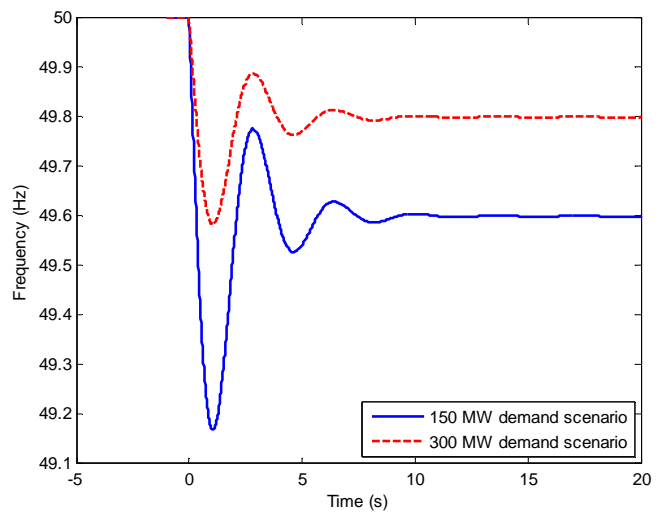

Figure 5: Frequency variation in case of generation tripping.

Table I: Comparison of the fundamental magnitudes of the response of a medium size isolated system.

\begin{tabular}{|l|l|l|}
\hline Demand scenario $(\mathrm{MW})$ & 150 & 300 \\
\hline Initial rate of change $(\mathrm{Hz} / \mathrm{s})$ & 1.33 & 0.67 \\
\hline Steady-state error $(\mathrm{Hz})$ & 0.4 & 0.2 \\
\hline Minimum frequency $(\mathrm{Hz})$ & 49.17 & 49.685 \\
\hline $\begin{array}{l}\text { Time of minimum frequency } \\
(\mathrm{s})\end{array}$ & 1.05 & 1.05 \\
\hline
\end{tabular}

The factor between the generation increase and the frequency decay $c$ is determined iteratively for this test system and applied to the computation of the minimum frequency and the time of minimum frequency. First, a time of minimum frequency $t_{\text {fmin }}$ is assumed, then the $c$ factor is computed and the time of minimum frequency $t_{\text {fmin }}$ is recomputed again. The process ends when the difference of $t_{f m i n}$ in two consecutive steps is smaller than tolerance. The $c$ factor in our system becomes:

$$
c=8.1395 \mathrm{pu}
$$

Table II provides the minimum frequency and the time of minimum frequency for this test system in case of a 30 MW generator tripping in the two demand scenarios of the minimum frequency and the time of minimum frequency using the approximate model of the frequency primary regulation. The comparison between Table I and Table II confirms the accuracy of the approximate mode of the frequency primary regulation.

Table II: Comparison of the fundamental magnitudes of the response of a medium size isolated system using a simplified model of the frequency primary regulation.

\begin{tabular}{|l|l|l|}
\hline Demand scenario (MW) & 150 & 300 \\
\hline Minimum frequency (Hz) & 49.386 & 49.693 \\
\hline $\begin{array}{l}\text { Time of minimum frequency } \\
\text { (s) }\end{array}$ & 0.922 & 0.922 \\
\hline
\end{tabular}

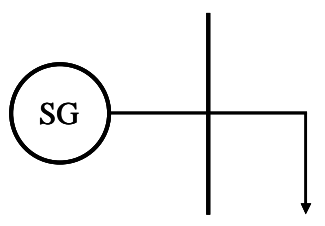

(I)

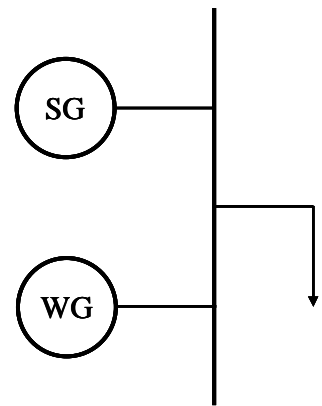

(II)
Figure 6: Test systems to evaluate the impact of wind power generation on the frequency stability of synchronous generators.

\section{$5 \quad$ Impact of Wind Generation on Frequency Stability}

Two simple test systems (see Figure 6) have been developed to evaluate the impact of wind power generators of the frequency stability of synchronous generators. Test system I contains a single synchronous generator (SG) of 200 MVA feeding a load of $160 \mathrm{Mw}$ and will be used as a reference. Test system II comprises of a synchronous and a wind power generator based on a doubly fed induction generator (DFIG) both of 100 MVA feeding a load. Such models include not only the models of the electrical machines but also the associated controls. The synchronous generator model includes models of the automatic voltage regulator and the speed governor-turbine system. 
DFIGs are the most widespread electromechanical energy conversion devices in wind power generation due their speed regulating capability. It allows them to operate in the optimal operating point (maximum efficiency) of the wind turbine. In addition, the network side power electronic converter provides them continuous reactive power compensation capability. Figure 7 displays the control scheme of a DFIG. The rotor windings are fed by a three-phase voltage source converter with pulse width modulation that provides a three-phase voltage system of variable frequency. The variation of the frequency of rotor currents results in a variation of the rotor speed. Assuming that the stator frequency $f_{1}$ is constant a variation of the rotor frequency $f_{2}$ results in a change of rotor speed $n$ according to:

$$
s=\frac{n_{1}-n}{n_{1}}=\frac{f_{2}}{f_{1}}
$$

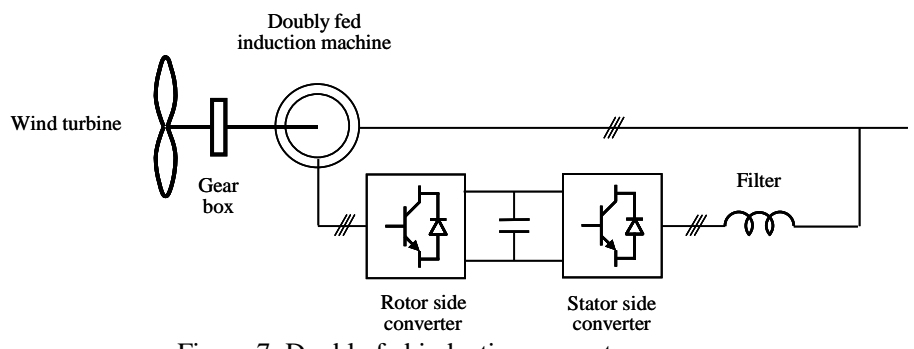

Figure 7: Doubly fed induction generator.

The electronic converter is built of two converters coupled through a dc link capacitor. The rotor converter is used to control either the torque or the rotor speed and the rotor reactive power. The component in the d-axis of the rotor current in a reference system solid with the stator flux is the excitation current and allows controlling the machine reactive power. The usual strategy is to set it to zero. The component in the q-axis of rotor current in a reference system solid with stator flux is the torque current and allows controlling the electromagnetic torque. The stator or network side converter is used to control the overall unit reactive power and the capacitor voltage. The component in the d-axis of the stator converter current determines the active power through the stator converter and allows controlling the capacitor voltage. The component in the q-axis of the stator converter current determines the reactive power provided by the stator converter.

We discuss the impact of DFIGs in the frequency stability of SGs [6]. Figure 8 compares the frequency deviation of the SG in test systems I and II in case of the trip of $20 \mathrm{MW}(10 \%$ of the system rating). The steady-state error of frequency when the system contains only a SG is $-0.04 \times 0.1=-0.004 \mathrm{pu}=-0.4 \%$. and when the system includes both a SG and a DFIG becomes $-0.04 \times 0.2=-0.008 \mathrm{pu}=-0.8 \%$. Not only the steady-state error of frequency changes due to the presence of a DFIG, but also the transient response is affected. Precisely, the initial rate-of-change of the frequency in the first case results $-0.1 / 6=-0.0167 \mathrm{pu} / \mathrm{s}=-1.67 \% / \mathrm{s}$ whereas in the second case becomes $-0.2 / 6=-0.033 \mathrm{pu} / \mathrm{s}=-3.33 \% / \mathrm{s}$. It means that the frequency variation is only determined by the inertia of the SG. In other words, the inertia of the DFIG does not affect to the frequency of the SG. Figure 9 also compares the mechanical power supplied by the SG in test systems I and II. It confirms that in both test systems the total generation tripped is supplied by the SG.

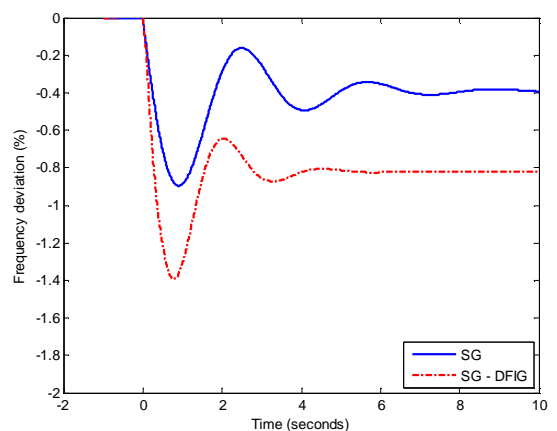

Figure 8: Comparison of the frequency deviation in systems with synchronous generators and in systems with synchronous and doubly fed induction generators.

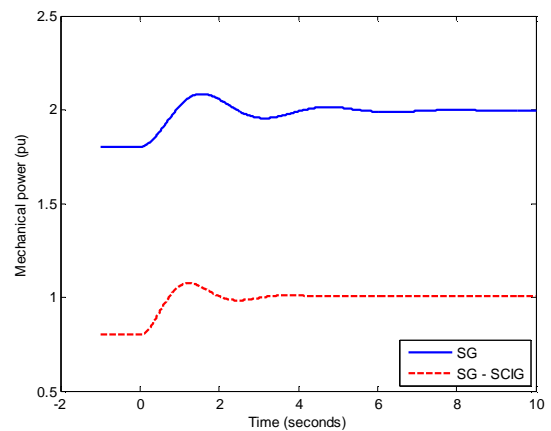

Figure 9: Comparison of the mechanical power supplied by the synchronous generator in systems with synchronous generators and in

systems with synchronous and doubly fed induction generators.

Wind power generation is limited by the fact that it does contribute to either the inertia or primary frequency regulation. We will determine first which is the maximum wind generation that is case of generator tripping does not result in load shedding because the frequency in below the setting of the underfrequency loadshedding relays.

The minimum frequency in case of a generator tripping is [7]:

$$
f_{\text {min }}=f_{0}\left(1-\frac{p_{G i, \max }}{2 \cdot c}\right)
$$

where $p_{G i \text {, max }}$ is the maximum generation tripping in per unit. If $p_{G i \text {,max }}$ is expressed in MW, equation (4) becomes:

$$
f_{\text {min }}=f_{0}\left(1-\frac{P_{G i, \max }}{2 \cdot c \cdot \frac{P_{L}+P_{R}-P_{W}}{\cos \varphi_{n}}}\right)
$$


being:

$S_{B}$ is the total system apparent power in MVA

$\cos \varphi_{n}$ is the nominal power factor of the generators

$P_{L}$ is the total load in $\mathrm{MW}$

$P_{R}$ is the total spinning reserve in MW

$P_{W}$ the total wind generation in MW

The maximum admissible wind generation is such that the minimum frequency $f_{\text {min }}$ is greater than the setting of the first stage of the underfrequency load shedding scheme $f_{\min }^{1}$. That is:

$$
f_{0}\left(1-\frac{P_{G i \text { max }}}{2 \cdot c \cdot \frac{P_{L}+P_{R}-P_{W}}{\cos \varphi_{n}}}\right) \geq f_{\text {min }}^{1}
$$

Hence

$$
P_{W} \leq P_{L}+P_{R}-\frac{P_{G i, \max } \cdot \cos \varphi_{n}}{2 \cdot c \cdot\left(1-\frac{f_{\min }^{1}}{f_{0}}\right)}
$$

Let us apply the relationship (5) to the test system discussed so far. It is a medium size system which fundamental features are:

- Minimum demand: $150 \mathrm{MW}$

- Maximum demand: $300 \mathrm{MW}$

- Biggest generator: $30 \mathrm{MW}$

- Spinning reserve: $30 \mathrm{MW}$

- Minimum frequency: $49 \mathrm{~Hz}$

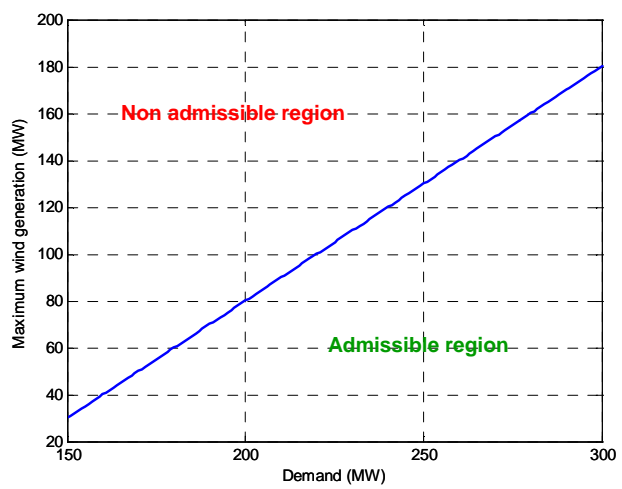

Figure 10: Maximum wind generation.

Figure 10 displays the admissible and non admissible regions in the plane total demand-maximum wind generation. For instance if the total demand is $220 \mathrm{MW}$, the wind generation should be lower than $80 \mathrm{MW}$ to prevent load shedding due to underfrequency in case than a $30 \mathrm{MW}$ generator (the largest unit in the system) is tripped. Figure 11 indicates how an energy storage system should be sized. Let us assume than the wind generation is $100 \mathrm{MW}$. If the total demand is greater than $220 \mathrm{MW}$, no energy storage system is required. However, if the total demand is smaller, an energy storage system is needed. Moreover, if the total demand is $200 \mathrm{MW}$, energy storage of $20 \mathrm{MW}$ must be incorporated.

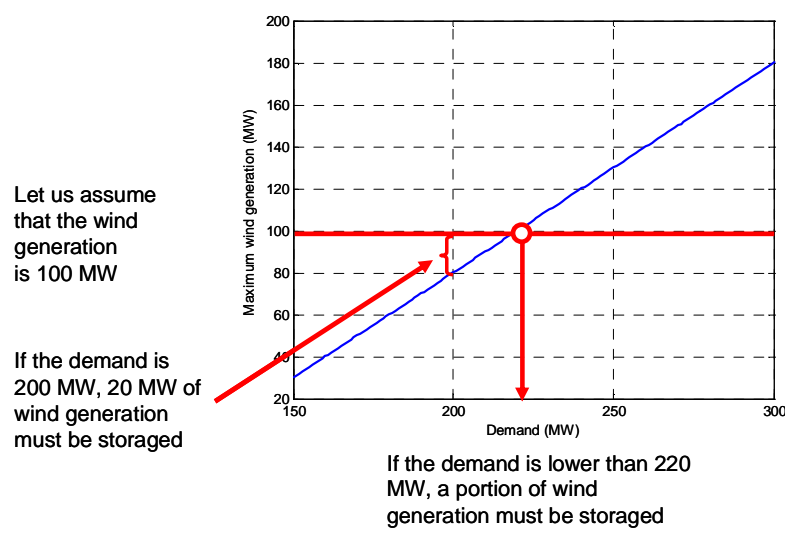

Figure 11: Sizing a storage system to overcome the limit of maximum wind generation.

\section{Conclusions}

High penetration of renewals may affect significantly frequency stability of isolated power systems since wind and solar photovoltaic generation have neither inertia nor primary frequency regulation. An alternative is the use of storage systems in the most critical operating conditions in such a way that if a generator trips, energy storage systems are subsequently tripped and the power balance is restored. This paper presents an approach to determine the maximum renewable generation in an isolated power system to prevent non-admissible frequency excursions in case of generator tripping. Moreover, such approach allows sizing energy storage systems to increase the penetration of renewals.

\section{References}

[1] P. Centeno, F. Fernández, L. Rouco, M. González, J.M. Rojo, J.R. Diago, "Estabilidad de los sistemas eléctricos insulares", Anales de Mecánica y Electricidad, Vol. LXXXI, Fascículo IV, Julio Agosto 2004, pp. 33 - 40.

[2] UCTE, "Operation handbook", available at http://www.ucte.org/resources/publications/ophandbook/.

[3] P. Kundur, "Power System Stability and Control", Mc Graw Hill, 1994.

[4] Red Eléctrica de España, "Procedimiento de Operación 1.6: Establecimiento de los planes de seguridad para la operación", available

http://www.ree.es/operacion/procedimientos operacion.asp.

[5] I. Egido, F. Fernández-Bernal, P. Centeno, L. Rouco, "Maximum Frequency Deviation Calculation in Small Isolated Power Systems", IEEE Transactions on Power Systems, Vol. 24, No. 4, pp. 1731-1738.

[6] L. Rouco, J. L. Zamora, I. Egido, F. Fernández, "Impact of wind power generation on the frequency stability of synchronous generators". Cigré Session 2008, Paper No. A1-203, Paris, 24-29 Agosto 2008

[7] I. Egido, L. Rouco, F. Fernández-Bernal, A. Rodríguez, J. Rupérez, F. Rodríguez-Bobada, S. Marín, "Máxima generación eólica en sistemas eléctricos aislados", XII ERIAC (Encuentro Regional Iberoamericano de Cigré), Foz de Iguazú, 20-24 Mayo 2007. 